\title{
Sequence set design for waveform-agile coherent radar systems
}

\author{
Jindong Zhang ${ }^{1 *}$, Naiqing $\mathrm{Xu}^{1}$, Hu Song ${ }^{2}$ and Chao Zhang ${ }^{1}$
}

\footnotetext{
*Correspondence: zjdjs@126.com 1 Nanjing University of Aeronautics and Astronautics, Nanjing, China Full list of author information is available at the end of the article
}

\begin{abstract}
With increased degrees of freedom of the transmitter, coherent waveform-agile radar system can change its transmission on-the-fly in response to target detection's requirement. This approach can provide better performance than a single waveform. In this paper, we consider unimodular sequence set design (USSD) problem based on summed range-Doppler ambiguity function (SRDAF) for coherent waveform-agile radar system. We present two algorithms for constructing unimodular sequence sets under the constant module constraint with desired minimized sidelobes on a predefined area of range-Doppler plane. The proposed algorithms are constructed based on singular value decomposition (SVD) and cyclic algorithm (CA), respectively. Numerical examples show the effectiveness of the proposed algorithms.
\end{abstract}

Keywords: Unimodular sequence set, Coherent radar, Waveform agility, Sidelobes, Optimization algorithm

\section{Introduction}

In the last decade, radar systems have been able to take full advantage of degrees of freedom of the receiver to improve performance. The emergence of prototype radar systems equipped with highly agile, software-driven waveform generators has provided the ability to change the transmit waveform at each time step to match environments and sensing objectives, such as increased signal-to-noise ratio (SNR), reduced estimation errors, or increased collection of information. Since there is an infinity of possible waveforms, it becomes critical to select or optimize the transit waveform at each time step. In coherent radar detection applications, the waveform-agile transmitter optimizes the waveform on a pulse-to-pulse basis and the radar system can obtain the information by optimally improving matched filter's response of the target.

In the literature, extensive research has been investigated single waveform design from the viewpoint of autocorrelation function and ambiguity function [1-25]. Some studies have also been focused on the choice of agile waveform in sensing. In the works [26-33], the dynamic selection of waveforms for target tracking was considered and the optimal waveform parameters were derived for tracking target motion using a linear/nonlinear observations model with detection in a clutter/clutter-free environment. These approaches can provide performance improvements over waveform optimization

(c) The Author(s). 2020 Open Access This article is licensed under a Creative Commons Attribution 4.0 International License, which permits use, sharing, adaptation, distribution and reproduction in any medium or format, as long as you give appropriate credit to the original author(s) and the source, provide a link to the Creative Commons licence, and indicate if changes were made. The images or other third party material in this article are included in the article's Creative Commons licence, unless indicated otherwise in a credit line to the material. If material is not included in the article's Creative Commons licence and your intended use is not permitted by statutory regulation or exceeds the permitted use, you will need to obtain permission directly from the copyright holder. To view a copy of this licence, visit http://creativecommons.org/licenses/by/4.0/. 
or improved tracking algorithm. Agile waveform can also be designed from the viewpoint of ambiguity function. The authors of [34] demonstrated that suitably transmitted and processed radar waveforms based on Golay sequences provide new primitives for adaptive waveform transmission. The adaptive transmission enables improved detection and finer resolution, while managing computational complexity at the receiver. Actually, Golay complementary sequences [35] can be seen as the simplest sequences in waveform-agile coherent radar system, and there is extensive literature because of the importance of such sequences in communications, coding theory, cryptology, and radar [36-41].

It should be noted that unimodular (i.e., constant modulus) sequences with good autocorrelation properties are useful in several areas, including communications and radar. The integrated sidelobe level (ISL) of the correlation function is often used to express the goodness of the correlation properties of a given sequence. In the references $[3,9,25]$, several cyclic algorithms for the local minimization of ISL-related metrics are presented. The power-like method are used for synthesizing sequence with good correlation and can also be extended for synthesizing sequences with good autocorrelation and cross-correlation functions in multiple-input multiple-output (MIMO) radar. However, these methods can not be directly utilized for optimizing sequences in waveform-agile coherent radar because they can only optimize range sidelobes level for correlation function.

In the aforementioned works, we have only considered ambiguity function synthesis for a single waveform, and little attention was paid to waveform design of coherent waveformagile radar based on summed range-Doppler ambiguity function (SRDAF). In addition, sidelobe shape control has also not been considered. In this paper, we investigate sequence set design problem with constant module, i.e., unimodular sequence set design (USSD) problem by SRDAF mathematical tool. Furthermore, we also consider that minimizing sidelobes on range-Doppler plane around the origin can improve the detection performance for closely spaced targets. We present two algorithms for constructing unimodular sequence sets under the constant module constraint with desired minimized sidelobes on a predefined area of range-Doppler plane. The proposed algorithms are constructed based on singular value decomposition (SVD) and cyclic algorithm (CA), respectively, and can be convergent by several hundreds of iterations.

In [42-44], coordinate descent (CD) algorithm is an important search algorithm and can solve optimization problems by successively performing approximate minimization along coordinate directions or coordinate hyperplanes. It has been used in applications for many years, and their popularity continues to grow because of their usefulness in data analysis, machine learning, and other areas of current interest. CD approach has been successfully applied for discrete-phase sequence design and minimum ISL code sequence design. In this paper, the performance of $C D$ approach is compared with the proposed algorithms.

The rest of this work is organized as follows. Section 2 discusses the mathematical model and ambiguity function of coherent waveform-agile radar system. Section 3 proposes two optimization algorithms for optimizing the shape of SRDAF based on SVD and CA, i.e., SVD-USSD and CA-USSD algorithms. Several numerical examples are presented in Section 4. Finally, concluding remarks and directions for future research are presented in Section 5. 


\section{Mathematical model and ambiguity function}

A radar system transmits a coherent pulse train consisting of $N$ pulses, which can be expressed as the following general form:

$$
s(t)=\sum_{n=0}^{N-1} s_{n}\left(t-n T_{r}\right),
$$

where $s_{n}(t)$ is the complex envelope of $n$th transmitted pulse and $T_{r}$ is the pulse repetition interval (PRI). We assume that $s_{n}(t)=0, \forall t \nsubseteq[0, T]$, where $T$ is the pulse duration. $s_{n}(t)$ is modulated by the following way

$$
s_{n}(t)=\sum_{m=0}^{M-1} x(n, m) p_{m}(t), \quad 0 \leq t \leq T,
$$

where $M$ is the subpluse length, $x(n, m), n=0,1, \ldots, N-1, m=0,1, \ldots, M-1$ is the modulating code sequence that is to be designed, and

$$
p_{m}(t)=\left\{\begin{array}{l}
\frac{1}{\sqrt{t_{p}}}, m t_{p} \leq t \leq(m+1) t_{p} \\
0, \quad \text { elsewhere, }
\end{array}\right.
$$

is an ideal rectangular shaping pulse of time length $t_{p}$ ( and thus $T=M t_{p}$ ). It is usually desired to transmit unimodular sequences

$$
x(n, m)=e^{j \phi_{n, m}},
$$

where $\left\{\phi_{n, m}\right\}$ are the phases. In common pulsed Doppler radar, the pulses with the same modulation, i.e., $s_{n}(t)=s_{0}(t), \forall n$, are transmitted consecutively in coherent processing interval (CPI). In this paper, we investigate the ambiguity function properties of waveform-agile pulsed Doppler radar.

The first processing step of pulsed Doppler is to implement matched filtering of the received echo. For a point target for $n$th pulse, the matched filter output can be given by

$$
\begin{aligned}
\chi_{n}(\tau, v)= & \int_{0}^{T} s_{n}(t) s_{n}(t-\tau) e^{j 2 \pi v\left(t-n T_{r}\right)} d t \\
= & e^{-j 2 \pi v n T_{r}} \sum_{k=0}^{M-1} \sum_{l=0}^{M-1} x^{*}(n, k) \\
& \cdot\left(\int_{0}^{T} p_{k}(t) p_{l}(t-\tau) e^{j 2 \pi v t} d t\right) x(n, l),
\end{aligned}
$$

where $\tau$ and $v$ are time delay and Doppler frequency shift of the target, respectively. We show that if, for all relevant Doppler frequency shift $-1 / 2 T_{r} \leq v \leq 1 / 2 T_{r}$, the pulse duration $T$ is small enough such that $|2 \pi \nu T|<\pi / 5$, which suggests $T \leq T_{r} / 5$, then the phase change in the received echo that is caused by the Doppler frequency of target motion can be neglected. Additionally, consider the time grid $\tau=p t_{p}, p=-(M-1), \ldots, 0, \ldots,(M-1)$ whose points are integer multiples of the subpulse length $t_{p}$. It is not difficult to calculate $\chi_{n}(\tau, v)$ at $\tau=p t_{p}$, which can be expressed as

$$
\begin{aligned}
\chi_{n}\left(p t_{p}, v\right)= & e^{j \pi t_{p}} e^{-j 2 \pi v n T_{r}} \frac{\sin \left(\pi v t_{p}\right)}{\pi v t_{p}} \\
& \cdot \sum_{m=0}^{M-1} x^{*}(n, m) x(n, m-p) .
\end{aligned}
$$


Note that $T_{r} \gg t_{p}$, and the Doppler unambiguity constraint $-1 / 2 T_{r} \leq v \leq 1 / 2 T_{r}$, we obtain

$$
\chi_{n}\left(p t_{p}, v\right) \approx e^{-j 2 \pi v n T_{r}} \sum_{m=0}^{M-1} x^{*}(n, m) x(n, m-p) .
$$

The second step for pulsed Doppler radar is to process the coherent pulse train, which is based on discrete Fourier transformation (DFT). To perform coherent processing of a particular delay, we must collect $N$ samples at a certain delay from last $N$ pulses. Therefore, we construct a two dimensional $(2 M-1) \times N$ array of matched filter output: first dimension is the delay, second dimension is the pulse number. The IDFT operation is then performed on $N$ samples of a row of the matched filter output, corresponding to a certain delay. The $p$ th IDFT processor output can be expressed as

$$
\xi(p, v)=\frac{1}{N} \sum_{n=0}^{N-1} e^{j 2 \pi v n T_{r}} \chi_{n}\left(p t_{p}, v\right)
$$

Consider the frequency grid $v=q / N T_{r}$, where $q=0,1, \ldots, N-1$. For convenience of expression, we ignore the constant in (8) and rewrite (8) as

$$
\xi(p, q)=\sum_{n=0}^{N-1} e^{j \frac{2 \pi}{N} n q} \chi_{n}\left(p t_{p}, v\right)
$$

It is no surprising that an ideal ambiguity function should have a high narrow peak in the origin and zero sidelobes everywhere else. Note that the target Doppler frequency $v$ only affect the high peak position in Doppler frequency axis, and the sidelobe energy is constant with all relevant $v$; thus, we can set $v=0$ and define a range-Doppler ambiguity function of pulse train, i.e. SRDAF, as

$$
\begin{aligned}
\xi(p, q) & =\sum_{n=0}^{N-1} \sum_{m=0}^{M-1} e^{j \frac{2 \pi}{N} n q} x^{*}(n, m) x(n, m-p) \\
& =\sum_{n=0}^{N-1} e^{j \frac{2 \pi}{N} n q} \rho_{n}(p),
\end{aligned}
$$

where $\rho_{n}(p)=\sum_{m=0}^{M-1} x^{*}(n, m) x(n, m-p)$ denotes the auto-correlation coefficient of the modulation code of the $n$th pulse.

It is easy to verify $\rho_{n}(0)=M, \forall n$. Thus, we have $\xi(0,0)=N M$, and $\xi(0, q)=0, q \neq 0$. Note that the shape of $\xi(p, q)$ is uncorrelated with the modulation codes $\{x(n, m)\}$ and $\xi(0,0)$, which indicates the peak value of SRDAF, is determined by the modulation code energy. Additionally, for $p \neq 0$, the shape of $\xi(p, q)$ is determined by the modulation codes $\{x(n, m)\}$ of $N$ pulses. Therefore, we can control the sidelobe of a a predefined area on range-Doppler plane by optimizing the modulation codes $\{x(n, m)\}$. For instance, the range and Doppler frequency of the observed target are a prior known by prescan or a prior information, and we can minimize the sidelobe of $\xi(p, q)$ in that area. Assume $I_{\Omega}$ is a subset including the positions of $(p, q)$ on range-Doppler plane for minimizing sidelobe 
and excluding positions on zero delay (i.e., $p=0$ ), the partial sidelobe minimization of $\xi(p, q)$ can be described by

$$
\begin{aligned}
\underset{\{x(n, m)\}}{\min } & \sum_{(p, q) \subset I_{\Omega}}|\xi(p, q)|^{2} \\
\text { s.t. } & |x(n, m)|=1 \\
& n=0,1, \ldots, N-1, m=0,1, \ldots, M-1 .
\end{aligned}
$$

Considering the objective function in (11) is a quartic form, which is difficult to tackle, we try to transform the above minimization problem into a quadratic form.

\section{Optimization method}

The optimization problem requires $N$ transmitted waveform $\left\{s_{n}(t)\right\}$ to be optimized. It is hard for us to optimize them simultaneously. By utilizing the alternating direction method, we can optimize the $n$th waveform $s_{n}(t)$ or its modulation code $\{x(n, m)\}_{m=0}^{M-1}$ while the others are fixed. Hence, the optimization problem in (11) can be transformed to $N$ optimization problems, and the $n$th problem can be given by

$$
\begin{aligned}
\mathcal{P}_{n}: \min _{n} & \sum_{(p, q) \subset I_{\Omega}}|\xi(p, q)|^{2} \\
\text { s.t. } & |x(n, m)|=1, \\
& m=0,1, \ldots, M-1 .
\end{aligned}
$$

where $\mathbf{x}_{n}=[x(n, 0) \quad x(n, 1) \quad \ldots \quad x(n, M-1)]^{T}$, and $(\cdot)^{T}$ denotes the transpose operation of a vector/matrix. In the following subsection, we will introduce an unimodular sequence set design method based on singular eigenvalue decomposition, namely SVD-USSD.

\subsection{SVD-USSD method}

By substituting (10) into (12), we optimize (12) by its autocorrelation coefficient $\left\{\rho_{n}(p)\right\}$ instead of $\mathbf{x}_{n}$. The corresponding optimization problem can be written as

$$
\widetilde{\mathcal{P}}_{n}: \min _{\rho_{n}} \sum_{(p, q) \subset I_{\Omega}}|\xi(p, q)|^{2} .
$$

Suppose that the sidelobe of AF shape on a region of range-Doppler plane is to be minimized, and $q(p) \subset\left[q_{p, \min }, q_{p, \max }\right]$, where $q_{p, \min }$ and $q_{p, \max }$ denote the bounds of the selected Doppler bins of the $p$ th range bin.

By further utilizing alternating direction method, the optimization problem $\widetilde{\mathcal{P}}_{n}$ can be split into multiple minimization problems, and the $p$ th sub optimization problem can be described by

$$
\widetilde{\mathcal{P}}_{n}^{(p)}: \min _{n}(p) \quad\left\|\mathbf{a}_{p} \rho_{n}(p)-\mathbf{b}_{p}\right\|^{2},
$$

where $\mathbf{a}_{p}=\left[\begin{array}{lll}e^{j 2 \pi n q_{p, \min } / N} & \ldots & e^{j 2 \pi n q_{p, \max } / N}\end{array}\right]^{T}$, and $\mathbf{b}_{p}=-\left[\sum_{k=0, k \neq n}^{N-1} e^{j 2 \pi k q_{p, \min } / N}\right.$ $\left.\rho_{k}(p) \quad \ldots \quad \sum_{k=0, k \neq n}^{N-1} e^{j 2 \pi k q_{p, \max } / N} \rho_{k}(p)\right]^{T},\|\cdot\|^{2}$ denotes the Frobenius matrix norm.

The optimal solution $\rho_{n}(p)$ of $\widetilde{\mathcal{P}}{ }_{n}^{(p)}$ can be easily given by the least square (LS) method:

$$
\rho_{n}(p)=\left(\mathbf{a}_{p}^{H} \mathbf{a}_{p}\right)^{(-1)} \mathbf{a}_{p}^{H} \mathbf{b}_{p}=\frac{1}{N_{p}} \mathbf{a}_{p}^{H} \mathbf{b}_{p},
$$


where $(\cdot)^{H}$ denotes the conjugate transpose operation, $N_{p}$ is the number of the selected Doppler bins of the $p$ th range bin.

Let

$$
\mathbf{X}_{n}=\left(\begin{array}{ccc}
x(n, 0) & & \\
\vdots & \ddots & \\
x(n, M-1) & & x(n, 0) \\
& \ddots & \vdots \\
& & x(n, M-1)
\end{array}\right)_{(2 M-1) \times M}
$$

With the obtained $\rho_{n}(p)$, we can construct a matrix, which is given by

$$
\mathbf{G}_{n}=\left(\begin{array}{cccc}
\rho_{n}(0) & \rho_{n}^{*}(1) & \cdots & \rho_{n}^{*}(M-1) \\
\rho_{n}(1) & \rho_{n}(0) & \ddots & \vdots \\
\vdots & \ddots & \ddots & \rho_{n}^{*}(1) \\
\rho_{n}(M-1) & \cdots & \rho_{n}(1) & \rho_{n}(0)
\end{array}\right)_{M \times M}
$$

Because $\mathbf{X}_{n}^{H} \mathbf{X}_{n}=\mathbf{G}_{n}$ indicates that the modulation code $\{x(n, m)\}_{m=0}^{M-1}$ is the optimal solution for (14), we can think of designing $\{x(n, m)\}_{m=0}^{M-1}$ by minimizing the following criterion

$$
\left\|\mathbf{X}_{n}^{H} \mathbf{X}_{n}-\mathbf{G}_{n}\right\|^{2}
$$

over the set of unimodular sequences. This optimization problem can be approximated by a simpler criterion

$$
\left\|\mathbf{X}_{n}-\mathbf{U G}_{n}^{1 / 2}\right\|^{2}
$$

where $\mathbf{U}$ is a $(2 M-1) \times M$ unitary matrix, i.e., $\mathbf{U}^{H} \mathbf{U}=\mathbf{I}$. The design problem associated with (19) can be stated as follows

$$
\begin{aligned}
& \widetilde{\mathcal{P} 2_{n}:} \begin{array}{r}
\{x(n, m)\}_{m=0}^{M-1} ; \mathbf{U}\left\|\mathbf{X}_{n}-\mathbf{U G}_{n}^{1 / 2}\right\|^{2} \\
\text { s.t. } \quad \mathbf{U}^{H} \mathbf{U}=\mathbf{I},
\end{array} \\
& |x(n, m)|=1, \quad m=0,1, \ldots, M-1 .
\end{aligned}
$$

Regarding the minimization problem in (20), we note the following facts. For given $\mathbf{X}_{n}$, let

$$
\mathbf{X}_{n} \mathbf{G}_{n}^{-1 / 2}=\mathbf{U}_{1} \Sigma \mathbf{U}_{2}^{H}
$$

denotes the SVD of $\mathbf{X}_{n} \mathbf{G}_{n}^{-1 / 2}$, where $\mathbf{U}_{1}$ is a $(2 M-1) \times M$ semi-unitary matrix, $\mathbf{U}_{2}$ is a $M \times M$ unitary matrix, and $\Sigma$ is a $M \times M$ diagonal matrix. Then, the solution of $\mathbf{U}$ for fixed $\mathbf{X}_{n}$ is given by

$$
\mathbf{U}=\mathbf{U}_{1} \mathbf{U}_{2}^{H} .
$$

Let $\mathbf{V}=\mathbf{U G}_{n}^{1 / 2}$, and $\left\{v_{m}\right\}_{m=0}^{M-1}$ are the elements of the matrix $\mathbf{V}$ whose positions are the same as the positions of $x(n, m)$ in $\mathbf{X}_{n}$. Then, it follows from (20) that the generic form of the minimization problem with respect to the elements of $\{x(n, m)\}_{m=0}^{M-1}$ is

$$
\min _{x(n, m)} \sum_{m=0}^{M-1}\left|x(n, m)-v_{m}\right|^{2} .
$$


Considering the unimodular constraint, the minimizer of (20) can be given by

$$
x(n, m)=e^{j \phi_{n, m}}, \quad \phi_{n, m}=\arg \left(\sum_{m=0}^{M-1} v_{m}\right) .
$$

The SVD-USSD method is summarized in Table 1.

Remark 1 In SVD-USSD optimization procedure, iterations are required to be performed and convergence can be obtained after a number of iterations. Note that eigenvalue decomposition (EVD) and SVD operations are performed once in every iteration step and hence the computational complexity of this algorithm is a little higher than expected. In practical occasions, where low complexity algorithms are more popular, algorithms without EVD and SVD operations can be preferable by radar system designer. In the next subsection, we will introduce a low complexity algorithm, i.e., CA-USSD algorithm.

\subsection{CA-USSD method}

The objective function in (12) is a quartic form, which is relatively difficult to obtain the global optimum by the analytical expression or the optimization method. In this section, we expect to find the local optimum for the problem in (28) and propose a computationally efficient approach.

Let $\mathbf{U}_{p, q}^{(n)}=e^{j 2 \pi n q / N} \mathbf{T}_{p}$, where $\mathbf{T}_{p}$ is a $M \times M$ shift matrix and defined as

$$
\mathbf{T}_{p}(k, l)=\left\{\begin{array}{l}
1, l=k+p \\
0, \text { elsewhere }
\end{array}\right.
$$

Table 1 The SVD-USSD algorithm

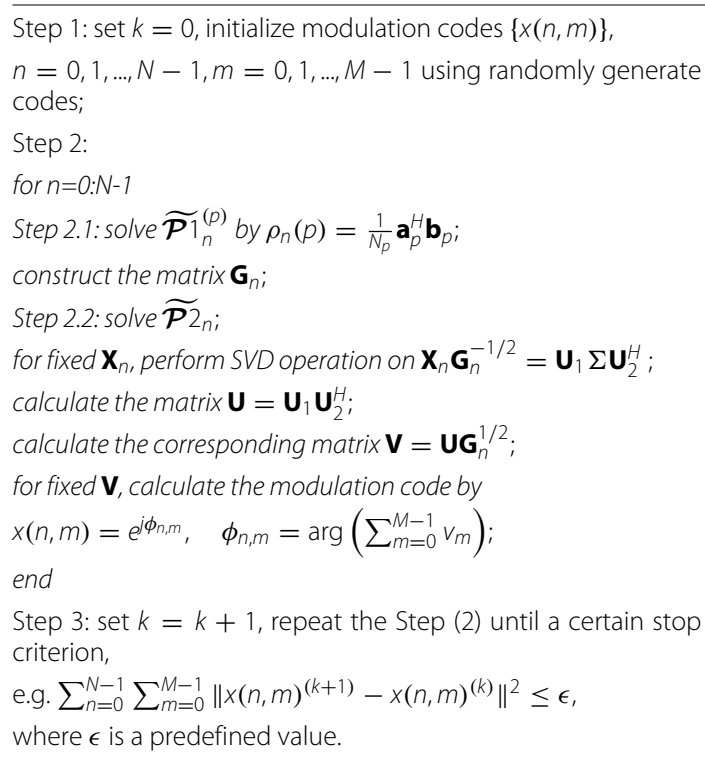


The objective function in (11) can be expressed as

$$
\begin{aligned}
\sum_{(p, q) \subset I_{\Omega}}|\xi(p, q)|^{2} & =\sum_{(p, q) \subset I_{\Omega}}\left|\sum_{n=0}^{N-1} \mathbf{x}_{n}^{H} \mathbf{U}_{p, q}^{(n)} \mathbf{x}_{n}\right|^{2} \\
& =\sum_{(p, q) \subset I_{\Omega}}\left|\mathbf{x}_{n}^{H} \mathbf{U}_{p, q}^{(n)} \mathbf{x}_{n}+\sum_{k=0, k \neq n}^{N-1} \mathbf{x}_{k}^{H} \mathbf{U}_{p, q}^{(k)} \mathbf{x}_{k}\right|^{2} \\
& =\mathbf{x}_{n}^{H} \mathbf{Q}_{n} \mathbf{x}_{n},
\end{aligned}
$$

where

$$
\mathbf{Q}_{n}=\sum_{(p, q) \subset I_{\Omega}}\left(\mathbf{U}_{p, q}^{(n) H}+\gamma_{p, q}^{(n)} \mathbf{I}_{M}\right)^{H} \mathbf{x}_{n} \mathbf{x}_{n}^{H}\left(\mathbf{U}_{p, q}^{(n) H}+\gamma_{p, q}^{(n)} \mathbf{I}_{M}\right)
$$

and

$$
\gamma_{p, q}^{(n)}=\frac{1}{M} \sum_{k=0, k \neq n}^{N-1} \mathbf{x}_{k}^{H} \mathbf{U}_{p, q}^{(k)} \mathbf{x}_{k}
$$

The optimization problem in (12) can be transformed to

$$
\begin{aligned}
\overline{\mathcal{P}}_{n}: \operatorname{\mathbf {x}}_{n} & \mathbf{x}_{n}^{H} \mathbf{Q}_{n} \mathbf{x}_{n} \\
\text { s.t. } & |x(n, m)|=1, \quad m=0,1, \ldots, M-1,
\end{aligned}
$$

where the $M \times M$ Hermitian matrix $\mathbf{Q}_{n}$ is positive definite.

Considering that the diagonal loading does not change the optimum solution of (29), this minimization problem can also be transformed to

$$
\begin{aligned}
\overline{\mathcal{P}}_{n}: \min _{n} & \mathbf{x}_{n}^{H} \widetilde{\mathbf{Q}}_{n} \mathbf{x}_{n} \\
\text { s.t. } & |x(n, m)|=1, \quad m=0,1, \ldots, M-1,
\end{aligned}
$$

where $\widetilde{\mathbf{Q}}_{n}=\mathbf{Q}_{n}-\lambda_{c} \mathbf{I}_{M}$ is a negative definite matrix if $\lambda_{c}>\lambda_{\max }\left(\mathbf{Q}_{n}\right)+1$. Here, $\lambda_{\max }(\cdot)$ denotes the largest eigenvalue, and we have $\widetilde{\mathbf{Q}}_{n}<-\mathbf{I}$.

In the following, we try to optimize (29) in a cyclic way. Let $\mathbf{x}_{n}^{(t)}$ and $\widetilde{\mathbf{Q}}_{n}^{(t)}$ be the obtained sequence and matrix at the $t$ th iteration.

Lemma 1 The following two inequalities

$$
\begin{gathered}
\mathbf{x}_{n}^{(t+1) H} \widetilde{\mathbf{Q}}_{n}^{(t+1)} \mathbf{x}_{n}^{(t+1)}<\mathbf{x}_{n}^{(t) H} \widetilde{\mathbf{Q}}_{n}^{(t+1)} \mathbf{x}_{n}^{(t)} \\
\mathbf{x}_{n}^{(t+1) H} \widetilde{\mathbf{Q}}_{n}^{(t)} \mathbf{x}_{n}^{(t+1)}<\mathbf{x}_{n}^{(t) H} \widetilde{\mathbf{Q}}_{n}^{(t)} \mathbf{x}_{n}^{(t)}
\end{gathered}
$$

can be obtained if the following condition is satisfied

$$
\begin{aligned}
& \operatorname{Re}\left[\mathbf{x}_{n}^{(t+1) H} \widetilde{\mathbf{Q}}_{n}^{(t+1)} \mathbf{x}_{n}^{(t)}\right]<\mathbf{x}_{n}^{(t) H} \widetilde{\mathbf{Q}}_{n}^{(t+1)} \mathbf{x}_{n}^{(t)}, \\
& \operatorname{Re}\left[\mathbf{x}_{n}^{(t+1) H} \widetilde{\mathbf{Q}}_{n}^{(t)} \mathbf{x}_{n}^{(t)}\right]<\mathbf{x}_{n}^{(t) H} \widetilde{\mathbf{Q}}_{n}^{(t)} \mathbf{x}_{n}^{(t)} .
\end{aligned}
$$

The proof of Lemma can be seen in [13].

Proof Because of the negative definiteness of the matrices $\widetilde{\mathbf{Q}}_{n}^{(t)}$ and $\widetilde{\mathbf{Q}}_{n}^{(t+1)}$, we can have

$$
\left(\mathbf{x}_{n}^{(t+1)}-\mathbf{x}_{n}^{(t)}\right)^{H} \widetilde{\mathbf{Q}}_{n}^{(t+1)}\left(\mathbf{x}_{n}^{(t+1)}-\mathbf{x}_{n}^{(t)}\right)<0
$$




$$
\left(\mathbf{x}_{n}^{(t+1)}-\mathbf{x}_{n}^{(t)}\right)^{H} \widetilde{\mathbf{Q}}_{n}^{(t)}\left(\mathbf{x}_{n}^{(t+1)}-\mathbf{x}_{n}^{(t)}\right)<0 .
$$

With the above two inequalities, we can obtain

$$
\begin{aligned}
\mathbf{x}_{n}^{(t+1) H} & \widetilde{\mathbf{Q}}_{n}^{(t+1)} \mathbf{x}_{n}^{(t+1)} \\
& <2 \operatorname{Re}\left\{\mathbf{x}_{n}^{(t+1) H} \widetilde{\mathbf{Q}}_{n}^{(t+1)} \mathbf{x}_{n}^{(t)}\right\}-\mathbf{x}_{n}^{(t) H} \widetilde{\mathbf{Q}}_{n}^{(t+1)} \mathbf{x}_{n}^{(t)},
\end{aligned}
$$

and

$$
\begin{aligned}
\mathbf{x}_{n}^{(t+1) H} & \widetilde{\mathbf{Q}}_{n}^{(t)} \mathbf{x}_{n}^{(t+1)} \\
& <2 \operatorname{Re}\left\{\mathbf{x}_{n}^{(t+1) H} \widetilde{\mathbf{Q}}_{n}^{(t)} \mathbf{x}_{n}^{(t)}\right\}-\mathbf{x}_{n}^{(t) H} \widetilde{\mathbf{Q}}_{m}^{(t)} \mathbf{x}_{n}^{(t)} .
\end{aligned}
$$

If $\operatorname{Re}\left[\mathbf{x}_{n}^{(t+1) H} \widetilde{\mathbf{Q}}_{n}^{(t+1)} \mathbf{x}_{n}^{(t)}\right]<\mathbf{x}_{n}^{(t) H} \widetilde{\mathbf{Q}}_{n}^{(t+1)} \mathbf{x}_{n}^{(t)}$ and $\operatorname{Re}\left[\mathbf{x}_{n}^{(t+1) H} \widetilde{\mathbf{Q}}_{n}^{(t)} \mathbf{x}_{n}^{(t)}\right]<\mathbf{x}_{n}^{(t) H} \widetilde{\mathbf{Q}}_{n}^{(t)} \mathbf{x}_{n}^{(t)}$ are satisfied at the same time, we can have

$$
\mathbf{x}_{n}^{(t+1) H} \widetilde{\mathbf{Q}}_{n}^{(t+1)} \mathbf{x}_{n}^{(t+1)}<\mathbf{x}_{n}^{(t) H} \widetilde{\mathbf{Q}}_{n}^{(t+1)} \mathbf{x}_{n}^{(t)},
$$

and

$$
\mathbf{x}_{n}^{(t+1) H} \widetilde{\mathbf{Q}}_{n}^{(t)} \mathbf{x}_{n}^{(t+1)}<\mathbf{x}_{n}^{(t) H} \widetilde{\mathbf{Q}}_{n}^{(t)} \mathbf{x}_{n}^{(t)}
$$

Note that $\mathbf{x}_{n}^{(t) H} \widetilde{\mathbf{Q}}_{n}^{(t+1)} \mathbf{x}_{n}^{(t)}=\mathbf{x}_{n}^{(t+1) H} \widetilde{\mathbf{Q}}_{n}^{(t)} \mathbf{x}_{n}^{(t+1)}$, therefore we can conclude

$$
\mathbf{x}_{n}^{(t+1) H} \widetilde{\mathbf{Q}}_{n}^{(t+1)} \mathbf{x}_{n}^{(t+1)}<\mathbf{x}_{n}^{(t) H} \widetilde{\mathbf{Q}}_{n}^{(t)} \mathbf{x}_{n}^{(t)}
$$

Remark 2 Lemma 1 indicates the requirement for the two inequalities, and it also states that the objective function $\mathbf{x}_{n}^{H(t)} \widetilde{\mathbf{Q}}_{n}^{(t)} \mathbf{x}_{n}^{(t)}$ is convergent and approaches the minimum value if (32) is satisfied. In the next step, the relationship between the above two inequalities in (31) is shown in Lemma 2.

Lemma 2 The two inequalities $\mathbf{x}_{n}^{(t) H} \widetilde{\mathbf{Q}}_{n}^{(t+1)} \mathbf{x}_{n}^{(t)}<\mathbf{x}_{n}^{(t) H} \widetilde{\mathbf{Q}}_{n}^{(t)} \mathbf{x}_{n}^{(t)}$ and $\mathbf{x}_{n}^{(t+1) H}$ $\widetilde{\mathbf{Q}}_{n}^{(t+1)} \mathbf{x}_{n}^{(t+1)}<\mathbf{x}_{n}^{(t) H} \widetilde{\mathbf{Q}}_{n}^{(t+1)} \mathbf{x}_{n}^{(t)}$ establish simultaneously if the sequence $\mathbf{x}_{n}^{(t+1)}$ can be obtained by the following minimization problem

$$
\underset{\mathbf{x}_{n}^{(t+1)}}{\min }\left\|\mathbf{x}_{n}^{(t+1)}+\widetilde{\mathbf{Q}}_{n}^{(t)} \mathbf{x}_{n}^{(t)}\right\|^{2} .
$$

Proof By expanding the objective function in (40), we obtain

$$
\left\|\mathbf{x}_{n}^{(t+1)}+\widetilde{\mathbf{Q}}_{n}^{(t)} \mathbf{x}_{n}^{(t)}\right\|^{2}=c+2 \operatorname{Re}\left\{\mathbf{x}_{n}^{(t+1) H} \widetilde{\mathbf{Q}}_{n}^{(t)} \mathbf{x}_{n}^{(t)}\right\}
$$

where $c=M^{2}+\left\|\widetilde{\mathbf{Q}}_{n}^{(t)} \mathbf{x}_{n}^{(t)}\right\|^{2}$ is a constant value. $\mathbf{x}_{n}^{(t+1)}$ is the minimizer of $\operatorname{Re}$ $\left\{\mathbf{x}_{n}^{(t+1) H} \widetilde{\mathbf{Q}}_{n}^{(t)} \mathbf{x}_{n}^{(t)}\right\}$. Hence, the second requirement can be satisfied by solving the minimization problem in (41). The solution for this problem can be easily given by

$$
\mathbf{x}_{n}^{(t+1)}=-\widetilde{\mathbf{Q}}_{n}^{(t)} \mathbf{x}_{n}^{(t)} .
$$


Considering the solution $\mathbf{x}_{n}^{(t+1)}=-\widetilde{\mathbf{Q}}_{n}^{(t)} \mathbf{x}_{n}^{(t)}$ and $\widetilde{\mathbf{Q}}_{n}^{(t)}<-\mathbf{I}$, we can obtain

$$
\begin{aligned}
\operatorname{Re} & {\left[\mathbf{x}_{n}^{(t+1) H} \widetilde{\mathbf{Q}}_{n}^{(t)} \mathbf{x}_{n}^{(t)}\right]-\mathbf{x}_{n}^{(t) H} \widetilde{\mathbf{Q}}_{n}^{(t)} \mathbf{x}_{n}^{(t)} } \\
& =-\mathbf{x}_{n}^{(t) H} \widetilde{\mathbf{Q}}_{n}^{(t) H} \widetilde{\mathbf{Q}}_{n}^{(t)} \mathbf{x}_{n}^{(t)}-\mathbf{x}_{n}^{(t) H} \widetilde{\mathbf{Q}}_{n}^{(t)} \mathbf{x}_{n}^{(t)} \\
& =\mathbf{x}_{n}^{(t) H}\left(-\widetilde{\mathbf{Q}}_{n}^{(t) H} \widetilde{\mathbf{Q}}_{n}^{(t)}-\widetilde{\mathbf{Q}}_{n}^{(t)}\right) \mathbf{x}_{n}^{(t)} \\
& =-\mathbf{x}_{n}^{(t) H}\left(\widetilde{\mathbf{Q}}_{n}^{(t) H}+\mathbf{I}\right) \widetilde{\mathbf{Q}}_{n}^{(t)} \mathbf{x}_{n}^{(t)}<0 .
\end{aligned}
$$

Considering the solution $\mathbf{x}_{n}^{(t+1)}=-\widetilde{\mathbf{Q}}_{n}^{(t)} \mathbf{x}_{n}^{(t)}$ and $\widetilde{\mathbf{Q}}_{n}^{(t+1)}<-\mathbf{I}$, we can also obtain

$$
\begin{aligned}
& \operatorname{Re}\left[\mathbf{x}_{n}^{(t+1) H} \widetilde{\mathbf{Q}}_{n}^{(t+1)} \mathbf{x}_{n}^{(t)}\right]-\mathbf{x}_{n}^{(t) H} \widetilde{\mathbf{Q}}_{n}^{(t+1)} \mathbf{x}_{n}^{(t)} \\
& =-\mathbf{x}_{n}^{(t) H} \widetilde{\mathbf{Q}}_{n}^{(t) H} \widetilde{\mathbf{Q}}_{n}^{(t+1)} \mathbf{x}_{n}^{(t)}-\mathbf{x}_{n}^{(t) H} \widetilde{\mathbf{Q}}_{n}^{(t+1)} \mathbf{x}_{n}^{(t)} \\
& =-\mathbf{x}_{n}^{(t) H}\left(\widetilde{\mathbf{Q}}_{n}^{(t) H}+\mathbf{I}\right) \widetilde{\mathbf{Q}}_{n}^{(t+1)} \mathbf{x}_{n}^{(t)}<0 .
\end{aligned}
$$

Considering the constant modulus constraint, the following operation can be added after (42)

$$
\widetilde{\mathbf{x}}_{n}^{(t+1)}=e^{j \arg \left(\mathbf{x}_{n}^{(t+1)}\right)},
$$

where $\arg (\cdot)$ denotes the argument of a complex number. The obtained unimodular sequence consists of different phase values, arbitrarily generated by $[0,2 \pi)$, and it is a suboptimal solution to (40).

The CA-USSD method is summarized in Table 2.

\section{Convergence analysis:}

The CA-USSD algorithm given in Table 2 is based on the general cyclic scheme; thus, according to Section 3.2, we know that the sequence of objective values evaluated at generated by the algorithm is nonincreasing. And it is easy to see that the objective value is bounded below by 0 ; thus, the sequence of objective values is guaranteed to converge to a finite value.

In this part, we will further analyze the convergence property of the sequence generated by the CA-USSD algorithm and show the convergence.

According to Lemma $1, \mathbf{x}_{n}^{(t+1) H} \widetilde{\mathbf{Q}}_{n}^{(t)} \mathbf{x}_{n}^{(t+1)}<\mathbf{x}_{n}^{(t) H} \widetilde{\mathbf{Q}}_{n}^{(t)} \mathbf{x}_{n}^{(t)}$ establishes if Re $\left[\mathbf{x}_{n}^{(t+1) H} \widetilde{\mathbf{Q}}_{n}^{(t)} \mathbf{x}_{n}^{(t)}\right]<\mathbf{x}_{n}^{(t) H} \widetilde{\mathbf{Q}}_{n}^{(t)} \mathbf{x}_{n}^{(t)}$. Therefore, $\mathbf{x}_{n}^{(t+1) H} \widetilde{\mathbf{Q}}_{n}^{(t)} \mathbf{x}_{n}^{(t+1)}<\mathbf{x}_{n}^{(t) H} \widetilde{\mathbf{Q}}_{n}^{(t)} \mathbf{x}_{n}^{(t)}$ also established if the equality $\mathbf{x}_{n}^{(t+1)}=-\widetilde{\mathbf{Q}}_{n}^{(t)} \mathbf{x}_{n}^{(t)}$ is satisfied.

Table 2 The CA-USSD algorithm

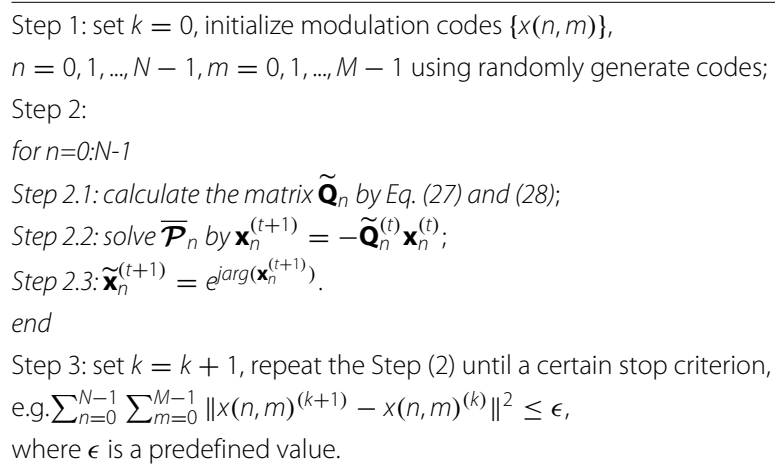


We can further note that

$$
\begin{aligned}
\mathbf{x}_{n}^{(t+1) H} & \widetilde{\mathbf{Q}}_{n}^{(t)} \mathbf{x}_{n}^{(t+1)}-\mathbf{x}_{n}^{(t) H} \widetilde{\mathbf{Q}}_{n}^{(t)} \mathbf{x}_{n}^{(t)} \\
& =\mathbf{x}_{n}^{(t) H} \widetilde{\mathbf{Q}}_{n}^{(t+1)} \mathbf{x}_{n}^{(t)}-\mathbf{x}_{n}^{(t) H} \widetilde{\mathbf{Q}}_{n}^{(t)} \mathbf{x}_{n}^{(t)}<0 \\
& =\mathbf{x}_{n}^{(t) H}\left(\widetilde{\mathbf{Q}}_{n}^{(t+1)}-\widetilde{\mathbf{Q}}_{n}^{(t)}\right) \mathbf{x}_{n}^{(t)}<0,
\end{aligned}
$$

and we have $\widetilde{\mathbf{Q}}_{n}^{(t+1)}<\widetilde{\mathbf{Q}}_{n}^{(t)}<-\mathbf{I}$.

We can also note that

$$
\begin{aligned}
\mathbf{x}_{n}^{(t+1) H} & \widetilde{\mathbf{Q}}_{n}^{(t+1)} \mathbf{x}_{n}^{(t+1)}-\mathbf{x}_{n}^{(t) H} \widetilde{\mathbf{Q}}_{n}^{(t+1)} \mathbf{x}_{n}^{(t)} \\
& =\mathbf{x}_{n}^{(t+1) H} \widetilde{\mathbf{Q}}_{n}^{(t+1)} \mathbf{x}_{n}^{(t+1)}-\mathbf{x}_{n}^{(t+1) H} \widetilde{\mathbf{Q}}_{n}^{(t)} \mathbf{x}_{n}^{(t+1)} \\
& =\mathbf{x}_{n}^{(t) H}\left(\widetilde{\mathbf{Q}}_{n}^{H(t)} \widetilde{\mathbf{Q}}_{n}^{(t+1)} \widetilde{\mathbf{Q}}_{n}^{(t)}-\widetilde{\mathbf{Q}}_{n}^{H(t)} \widetilde{\mathbf{Q}}_{n}^{(t)} \widetilde{\mathbf{Q}}_{n}^{(t)}\right) \mathbf{x}_{n}^{(t)} \\
& =\mathbf{x}_{n}^{(t) H} \widetilde{\mathbf{Q}}_{n}^{H(t)}\left(\widetilde{\mathbf{Q}}_{n}^{(t+1)}-\widetilde{\mathbf{Q}}_{n}^{(t)}\right) \widetilde{\mathbf{Q}}_{n}^{(t)} \mathbf{x}_{n}^{(t)},
\end{aligned}
$$

and verify the negative definiteness of the matrix $\widetilde{\mathbf{Q}}_{n}^{H(t)}\left(\widetilde{\mathbf{Q}}_{n}^{(t+1)}-\widetilde{\mathbf{Q}}_{n}^{(t)}\right) \widetilde{\mathbf{Q}}_{n}^{(t)}$ by $\widetilde{\mathbf{Q}}_{n}^{(t+1)}<$ $\widetilde{\mathbf{Q}}_{n}^{(t)}$, thus $\mathbf{x}_{n}^{(t+1) H} \widetilde{\mathbf{Q}}_{n}^{(t+1)} \mathbf{x}_{n}^{(t+1)}<\mathbf{x}_{n}^{(t) H} \widetilde{\mathbf{Q}}_{n}^{(t+1)} \mathbf{x}_{n}^{(t)}$ can also exists if $\mathbf{x}_{n}^{(t+1)}=-\widetilde{\mathbf{Q}}_{n}^{(t)} \mathbf{x}_{n}^{(t)}$.

The results of (45) and (46) highlight that if the equality $\mathbf{x}_{n}^{(t+1)}=-\widetilde{\mathbf{Q}}_{n}^{(t)} \mathbf{x}_{n}^{(t)}$ is satisfied, then the inequalities $\mathbf{x}_{n}^{(t+1) H} \widetilde{\mathbf{Q}}_{n}^{(t)} \mathbf{x}_{n}^{(t+1)}<\mathbf{x}_{n}^{(t) H} \widetilde{\mathbf{Q}}_{n}^{(t)} \mathbf{x}_{n}^{(t)}$ and $\mathbf{x}_{n}^{(t+1) H} \widetilde{\mathbf{Q}}_{n}^{(t+1)}$ $\mathbf{x}_{n}^{(t+1)}<\mathbf{x}_{n}^{(t) H} \widetilde{\mathbf{Q}}_{n}^{(t+1)} \mathbf{x}_{n}^{(t)}$ are correspondingly obtained. Therefore, the objective function $\mathbf{x}_{n}^{(t) H} \widetilde{\mathbf{Q}}_{n}^{(t)} \mathbf{x}_{n}^{(t)}$ is convergent if the sequence satisfy $\mathbf{x}_{n}^{(t+1)}=-\widetilde{\mathbf{Q}}_{n}^{(t)} \mathbf{x}_{n}^{(t)}$ in iteration procedure. Due to iterative calculations, the obtained sequence $\mathbf{x}_{n}^{(t)}$ usually does not satisfy the constant modulus constraint.

\subsection{Computational complexity}

In the SVD-USSD procedure, the computational complexity of $\mathbf{G}_{n}^{-1 / 2}$ can be performed by the eigenvalue decomposition (EVD) at complexity order of $\mathcal{O}\left(26 M^{3}\right)$, and the computational complexity of $\mathbf{U}=\mathbf{U}_{1} \mathbf{U}_{2}^{H}$ can be performed by the singular value decomposition (SVD) at complexity order of $\mathcal{O}\left(4 M(M-1)^{2}+22 M^{3}\right)$. Thus, the overall computational complexity of the SVD-USSD algorithm $\mathcal{O}\left(K\left(26 M^{3}+4 M(M-1)^{2}+22 M^{3}+(6 M-2) M^{2}\right)\right)$, where $K$ is the total number of iterations. In the CA-USSD procedure, the overall computational complexity is $\mathcal{O}\left(K L\left(4 M^{2}+2 M\right)\right)$, where $L$ is the number of the selected range and Doppler bins. It is obvious that the computational complexity of CA-USSD algorithm is obviously lower than that of SVD-USSD algorithm.

\section{Simulation results}

In this section, we provide several simulation examples to demonstrate the performance of the proposed methods, SVD-USSD and CA-USSD. In the following examples, it is assumed that the radar pulse width $T$ is 10 us, and the PRI equals to $1 \mathrm{~ms}$ to ensure the Doppler frequency insensitivity of the transmit waveform. The coherent radar system transmits $N=64$ pulses in a CPI and $M=100$ subpulses in a pulse. To evaluate the shape of SRDAF of the waveform-agile radar system, a point-like target is considered in the simulations. In SRDAF image, the time delay axis is normalized by subpulse duration $t_{p}$, and the Doppler frequency shift axis is normalized by $T_{r}$. The convergence of the proposed algorithms will be tested by using randomly generated sequences in the initialization.

In this example, the shape of SRDAF is desired to have sidelobes in an interested area as low as possible. Therefore, we suppose that $\Omega=\{(p, q)|| q \mid \leq N / 4,(p, q) \neq(0,0)\}$ is the 
area on range-Doppler plane, which is close to the origin but excludes the origin. With randomly generated sequences in the initialization, the proposed algorithms in Section 3 is utilized to minimized the ISL of the SRDAF of the synthesized sequences.

The shape of SRDAF of sequence set optimized by SVD-USSD and CA-USSD algorithms are shown in Fig. 1a and b. The sidelobes of the SRDAFs of the sequence sets synthesized by SVD-USSD and CA-USSD algorithms are suppressed to about - $50 \mathrm{~dB}$ in the interested area on range-Doppler plane . In Fig. 1c, the curves of the objective functions vs. iteration number in SVD-USSD and CA-USSD algorithms are given. The convergence performance of these two algorithms are almost the same and can converge to a stable point after 300 iterations. However, SVD-USSD algorithm shows faster convergence speed.

To evaluate the sidelobe synthesis performances of the proposed SVD-USSD and CAUSSD algorithms, we define the average sidelobe level and peak sidelobe level by

$$
\mathrm{ASL}=20 \log _{10} \frac{\xi(0,0)}{\sqrt{\frac{1}{N_{\Omega}} \sum_{(p, q) \subset I_{\Omega}}|\xi(p, q)|^{2}}}(\mathrm{~dB})
$$

, and

$$
\mathrm{PSL}=20 \log _{10} \frac{\xi(0,0)}{\max _{(p, q) \subset I_{\Omega}}|\xi(p, q)|}(\mathrm{dB})
$$

Figure 2 plots the curves of average sidelobe level vs. sequence length and sequence number of the sequence set optimized by SVD-USSD and CA-USSD algorithms after 200 iterations, i.e., average sidelobe level vs. sequence length $M$, average sidelobe level vs. sequence number $N$, peak sidelobe level vs. sequence length $M$, and peak sidelobe level vs. sequence number $N$, respectively. As sequence length $M$ increases from 20 to 100 and sequence number $N$ equals to 64 , ASL is decreased from $-40 \mathrm{~dB}$ to $-48 \mathrm{~dB}$ for CA-USSD algorithm and decreased from $-44 \mathrm{~dB}$ to $-51 \mathrm{~dB}$ for SVD-USSD algorithm. Meanwhile, PSL is decreased from $-57 \mathrm{~dB}$ to $-68 \mathrm{~dB}$ for CA-USSD algorithm and decreased from $-62 \mathrm{~dB}$ to $-75 \mathrm{~dB}$ for SVD-USSD algorithm.

As sequence number $M$ increases from 10 to 130 and sequence length $M$ equals to 100, ASL is decreased from $-36 \mathrm{~dB}$ to $-48 \mathrm{~dB}$ for CA-USSD algorithm, and ASL is decreased from $-40 \mathrm{~dB}$ to $-55 \mathrm{~dB}$ for SVD-USSD algorithm. Meanwhile, PSL is decreased from $-52 \mathrm{~dB}$ to $-72 \mathrm{~dB}$ for CA-USSD algorithm and decreased from $-59 \mathrm{~dB}$ to $-79 \mathrm{~dB}$ for SVD-USSD algorithm.

In the simulation result of Fig. 2, CD approach is utilized for synthesizing unimodular sequence set with 4PSK, 16PSK, and 64PSK modulation when the difference of objective function between two adjacent iterations is small enough. In the iteration process, the parameter $\epsilon$ is set to be $10^{-4}$. Due to the limited number of discrete phases, the ISL and PSL performance of CD-4PSK, CD-16PSK, and CD-64PSK is higher than that of SVDUSSD and CA-USSD algorithms. However, with increased number of discrete phases, the ISL and PSL performance is improved. The performance of CD-64PSK is close to that of SVD-USSD and CA-USSD algorithms. In Fig. $2 b$ and d, we can also find that the performance of CD-64PSK is even better with small sequence number $N$.

In Tables 3 and 4, runtime of SVD-USSD and CA-USSD is given after 200 iterations. Runtime of the CD-USSD algorithm is also given with 16PSK modulation. CA-USSD algorithm has the shortest running time, CD-USSD has the longest running time and 


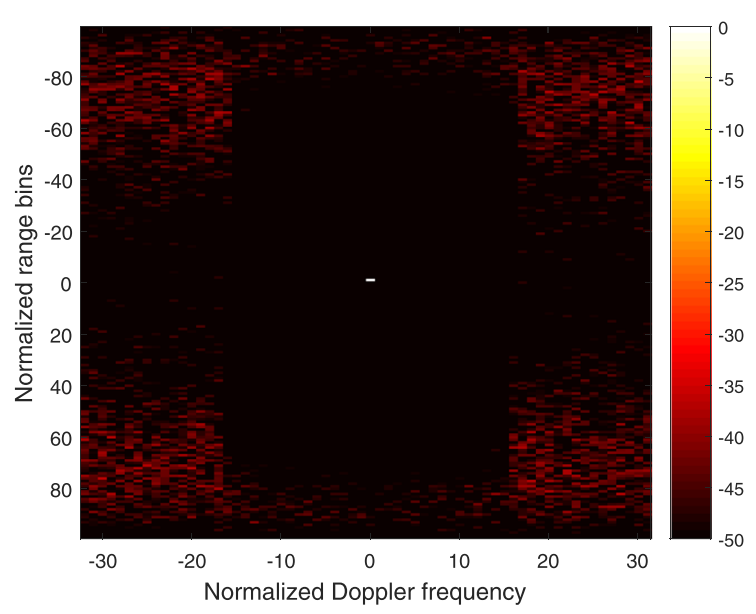

(a)

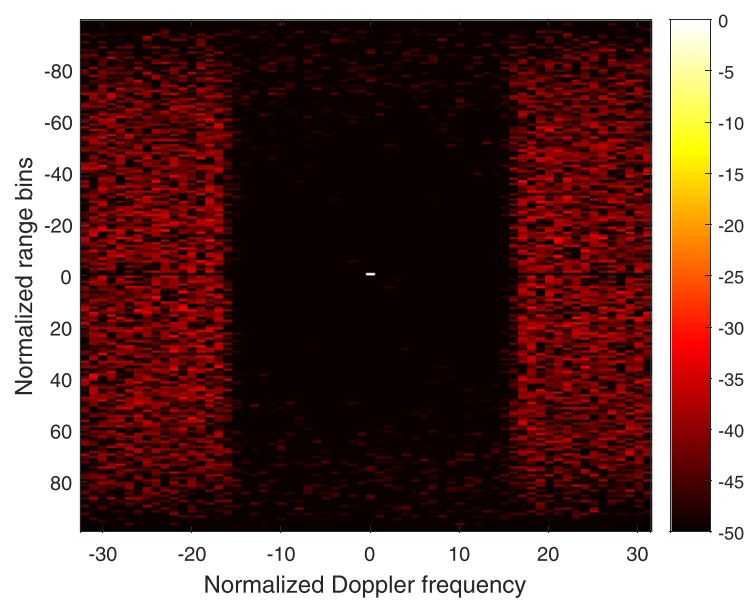

(b)

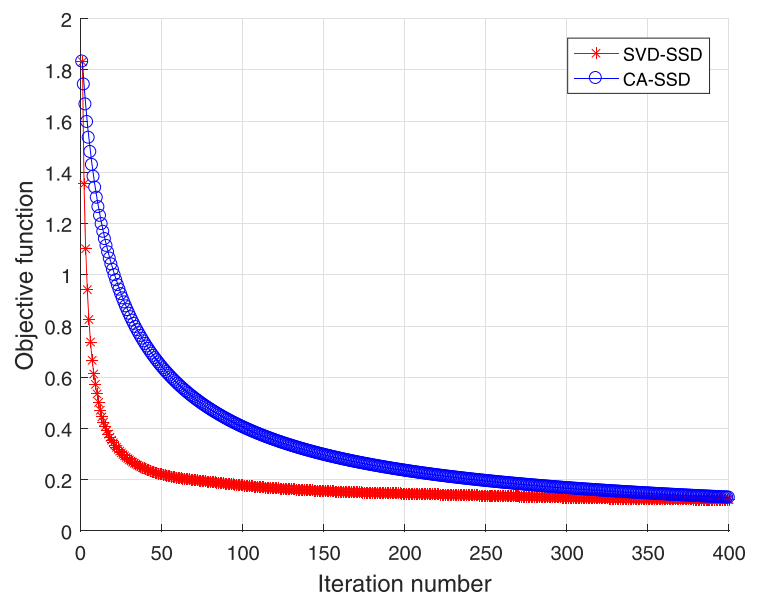

(c)

Fig. 1 The shape of SRDAF of sequence set optimized by SVD-USSD and CA-USSD algorithms, a SVD-USSD algorithm, b CA-USSD algorithm, $\mathbf{c}$ the curve of the objective function vs. iteration number of SVD-USSD and CA-USSD algorithms 


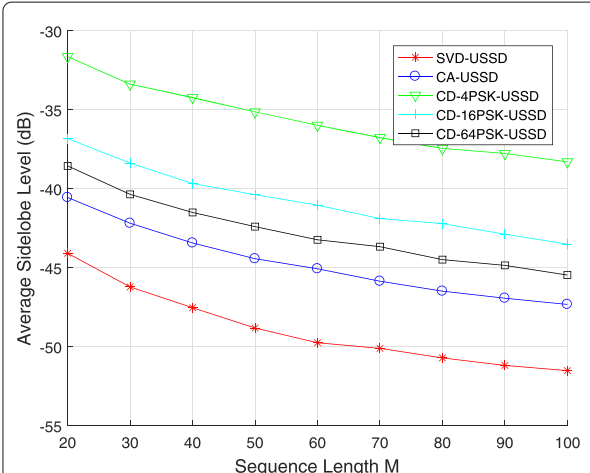

(a)

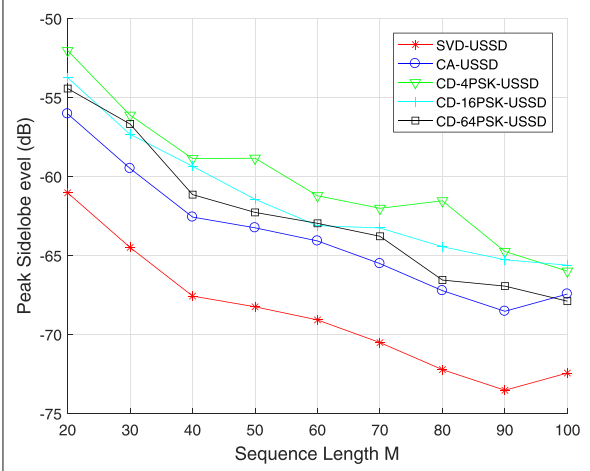

(c)

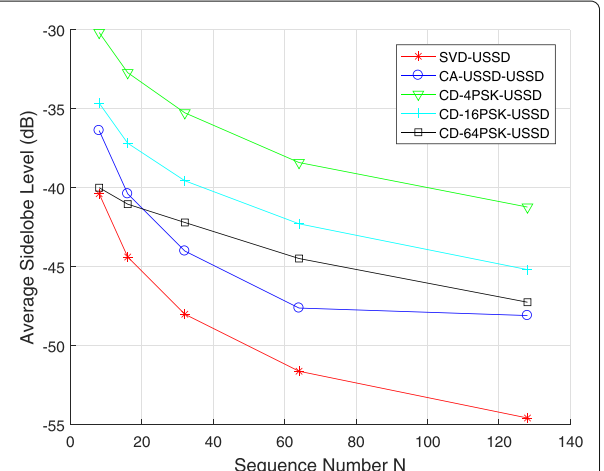

(b)

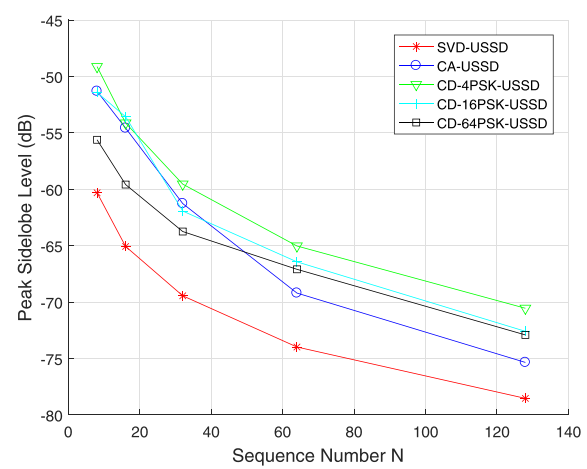

(d)

Fig. 2 Curve of average sidelobe level vs. sequence length and sequence number of the sequence set optimized by SVD-USSD, CA-USSD, and CD algorithms, a ASL vs. M, b ASL vs. N, c PSL vs. M, and d PSL vs. N

SVD-USSD algorithm has the middle running time. We also note that SVD-USSD algorithm can give better optimization results than CA-USSD algorithm because of faster convergence speed. Although the proposed two algorithms can achieve almost the same optimization performance after 300 iterations, SVD-USSD algorithm can obtain better performance under fewer iteration condition.

Figure 3 shows the range-Doppler processing results by using Golomb sequence, random sequences and sequence sets optimized by SVD-USSD and CA-USSD algorithms. It is assumed that there exists five scattering points, which are located at $(0,0),(20,7.5),(20,-7.5),(-20,7.5)$, and $(-20,-7.5)$ on range-Doppler plane. As we can see, two scattering points are emerged by the sidelobes of the range-Doppler processing result of Golomb sequence. The sidelobes of the range-Doppler processing result of random sequences is lower than that of Golomb sequence. With sequence sets optimized

Table 3 Runtime of SVD-USSD, CA-USSD, and CD-USSD algorithms

\begin{tabular}{llll}
\hline Method & SVD-USSD & CA-USSD & CD-USSD (16PSK) \\
\cline { 1 - 3 }$M=100$ & & & $189 \mathrm{~s}$ \\
\hline$N=8$ & $92.6 \mathrm{~s}$ & $16.4 \mathrm{~s}$ & $1245 \mathrm{~s}$ \\
$N=16$ & $215.3 \mathrm{~s}$ & $35.8 \mathrm{~s}$ & $8447 \mathrm{~s}$ \\
$N=32$ & $350.6 \mathrm{~s}$ & $62.6 \mathrm{~s}$ & $57,639 \mathrm{~s}$ \\
$N=64$ & $731.1 \mathrm{~s}$ & $122.1 \mathrm{~s}$ & $48,065 \mathrm{~s}$ \\
$N=128$ & $1382.3 \mathrm{~s}$ & $252.4 \mathrm{~s}$ & \\
\hline
\end{tabular}


Table 4 Runtime of SVD-USSD, CA-USSD, and CD-USSD algorithms

\begin{tabular}{llll}
\hline Method & SVD-USSD & CA-USSD & CD-USSD (16PSK) \\
\cline { 1 - 3 } $\mathrm{N}=64$ & & & \\
\hline$M=20$ & $5.9 \mathrm{~s}$ & $5.4 \mathrm{~s}$ & $589 \mathrm{~s}$ \\
$M=40$ & $47.3 \mathrm{~s}$ & $21.2 \mathrm{~s}$ & $4255 \mathrm{~s}$ \\
$M=60$ & $159.9 \mathrm{~s}$ & $47.4 \mathrm{~s}$ & $18325 \mathrm{~s}$ \\
$M=80$ & $379.2 \mathrm{~s}$ & $84.1 \mathrm{~s}$ & $32844 \mathrm{~s}$ \\
$M=100$ & $741.1 \mathrm{~s}$ & $131.2 \mathrm{~s}$ & $65429 \mathrm{~s}$ \\
\hline
\end{tabular}

by SVD-USSD and CA-USSD algorithms, sidelobes is obviously suppressed and the best detection performance can be obtained.

\section{Discussions}

It should be noted that CA-USSD algorithm avoids EVD and SVD operations in iteration procedure, and the computational complexity of this algorithm is obviously lower than that of SVD-USSD algorithm. However, it does not mean that CA-USSD algorithm outperforms SVD-USSD algorithm in every aspect. In the above section, we compare the two algorithms thoroughly. SVD-USSD algorithm has higher computational complexity and better optimization result. The computational complexity of CA-USSD algorithm is lower, but the optimization result of CA-USSD algorithm is not as good as that of SVD-USSD algorithm.

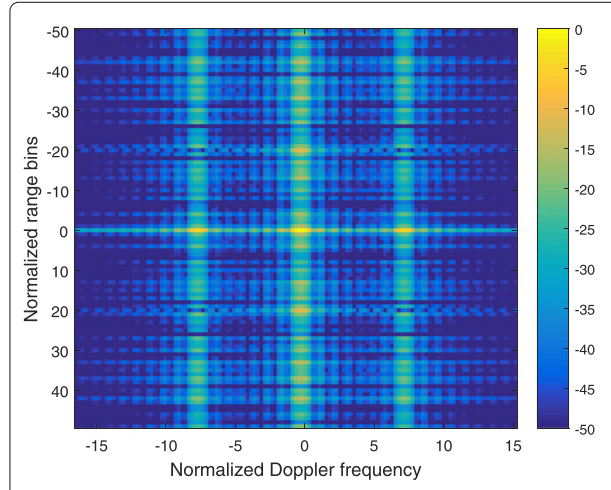

(a)

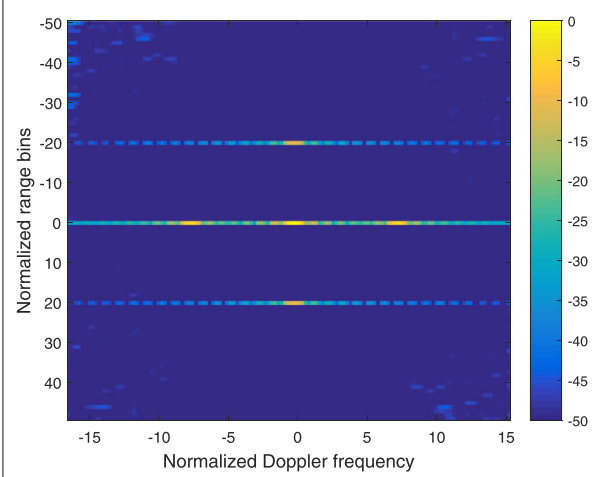

(c)

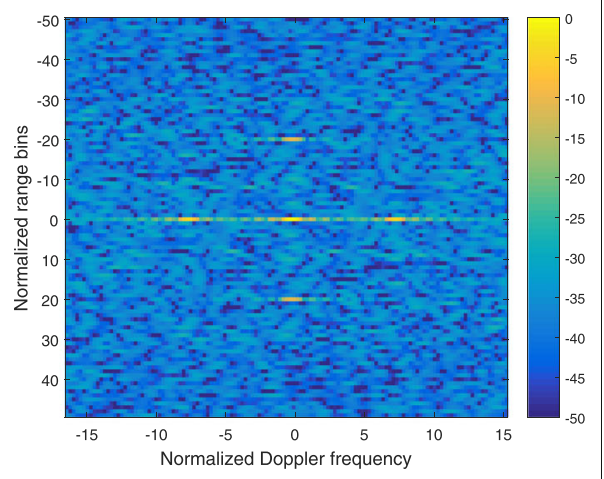

(b)

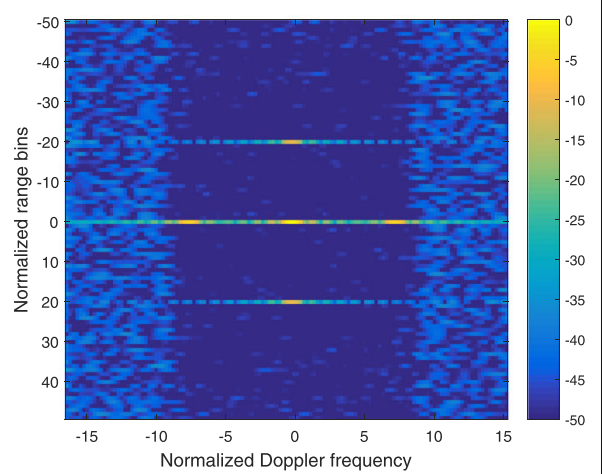

(d)

Fig. 3 Range-Doppler processing results by using Golomb sequence, random sequences, and sequence set optimized by SVD-USSD and CA-USSD algorithms: a Golomb sequence, $\mathbf{b}$ random sequences, $\mathbf{c}$ sequence set optimized by SVD-USSD algorithm, and $\mathbf{d}$ sequence set optimized by CA-USSD algorithm 


\section{Conclusion}

In this paper, two algorithms, i.e., SVD-USSD and CA-USSD, for constructing unimodular sequence sets under constant module constraint with desired minimized sidelobes on a predefined area of range-Doppler plane are presented. SVD-USSD and CA-USSD algorithms show their advantages in different aspect. SVD-USSD algorithm has higher computational complexity and better optimization result. The computational complexity of CA-USSD algorithm is lower, but the optimization result of CA-USSD is not as good as that of SVD-USSD.

We further note that the computational efficiency of SVD-USSD limited by SVD operation. This algorithm is better for the sequences of the length no longer than $10^{4}$. Although the convergence speed of CA-USSD is slower than that of SVD-USSD, computational efficiency of CA-USSD is better and more suitable for the sequences with longer length.

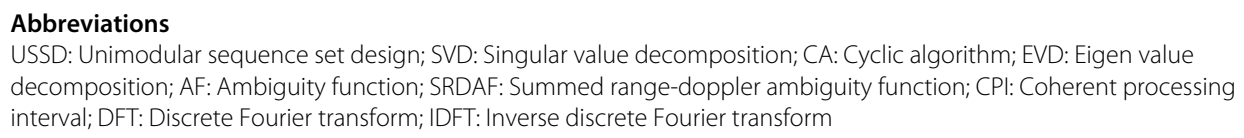

\section{References}

1. F. Gini, A. De Maio, L. Patton, Waveform design and diversity for advanced radar systems. (IET Radar and Sonar Navigation, London, 2011)

2. A. Aubry, A. De Maio, M. M. Naghsh, Optimizing radar waveform and Doppler filter bank via generalized fractional programming. IEEE J. Sel. Top. Sig. Proc. 9(8), 1387-1399 (2015)

3. M. A. Kerahroodi, A. Aubry, A. De Maio, M. M. Naghsh, M. Modarres-Hashemi, A coordinate-descent framework to design low PSL/ISL sequences. IEEE Trans. Sig. Proc. 65(22), 5942-5956 (2017)

4. N. Zhang, S. W. Golomb, Polyphase sequence with low autocorrelations. IEEE Trans. Inf. Theory. 39(3), 1085-1089 (1993)

5. R. Frank, Polyphase codes with good nonperiodic correlation properties. IEEE Trans. Inf. Theory. 9(1), $43-45$ (1963)

6. C. D. Groot, D. Wurtz, K. H. Hoffmann, Low autocorrelation binary sequences: exact enumeration and optimization by evolutionary strategies. Optimization. 23(4), 369-384 (1992)

7. H. D. Schotten, H. D. Liß̧uke, On the search for low correlated binary sequences. Int. J. Electron. Commun. 59(2), 67-78 (2005)

8. S. Mertens, Exhaustive search for low-autocorrelation binary sequences. Phys. J. A. 29, 473-481 (1996) 
9. P. Stocia, H. He, J. Li, New algorithms for designing unimodular sequences with good correlation properties. IEEE Trans. Sig. Proc. 57(4), 1415-1425 (2009)

10. J. Li, P. Stoica, X. Zheng, Signal synthesis and receiver design for MIMO radar imaging. IEEE Trans. Sig. Proc. 56(8), 3959-3968 (2008)

11. M. Soltanalian, P. Stoica, Computational design of sequences with good correlation properties. IEEE Trans. Sig. Proc. 60(5), 2180-2193 (2012)

12. P. Stoica, H. He, J. Li, On designing sequences with impulse-like periodic correlation. IEEE Trans. Sig. Proc. 16(8), 703-706 (2009)

13. M. Soltanalian, P. Stoica, Designing unimodular codes via quadratic optimization. IEEE Trans. Sig. Proc. 62(5) (2014). https://doi.org/10.1109/tsp.2013.2296883

14. S. Sussman, Least-square synthesis of radar ambiguity functions. IEEE Trans. Inf. Theory. 8(3), 246-254 (1962)

15. J. D. Wolf, G. M. Lee, C. E. Suyo, Radar waveform synthesis by mean-square optimization techniques. IEEE Trans. Aerosp. Electron. Syst. 5(4), 611-619 (1968)

16. I. Gladkova, D. Chebanov, in International Conference on Radar Systems, On the synthesis problem for a waveform having a nearly ideal ambiguity functions (France, Toulouse, 2004)

17. Y. I. Abramovich, B. G. Danilov, A. N. Meleshkevich, Application of integer programming to problems of ambiguity function optimization. Radio Eng. Elect. Phys. 22(5), 48-52 (1977)

18. A. Aubry, A. De Maio, B. Jiang, Ambiguity function shaping for cognitive radar via complex quartic optimization. IEEE Trans. Sig. Proc. 61(22), 5603-5619 (2013)

19. M. Soltanalian, B. Tang, J. Li, et al., Joint design of the receive filter and transmit sequence for active sensing. IEEE Sig. Proc. Lett. 20(5), 423-426 (2013)

20. H. He, P. Stocia, in IEEE International Conference on Acoustics, Speech and Signal Processing (ICASSP), On synthesizing cross ambiguity functions, (Prague, 2011), pp. 3536-3539. https://doi.org/10.1109/icassp.2011.5946241

21. P. Stoica, H. He, J. Li, Optimization of the receive filter and transmit sequence for active sensing. IEEE Trans. Sig. Proc. 60(4), $1730-1740$ (2012)

22. A. Aubry, A. Demaio, A. Farina, M. Wicks, Knowledge-aided (potentially cognitive) transmit signal and receive filter design in signal-dependent clutter. IEEE Trans. Aerosp. Electron. Syst. 49(1), 93-117 (2013)

23. S. Kay, Optimal signal design for detection of Gaussian point targets in stationary Gaussian clutter/reverberation. IEEE J. Sel. Top. Sig. Proc. 1(1), 31 -41 (2007)

24. A. Aubry, et al., Cognitive design of the receive filter and transmitted phase code in reverberating environment. IET Radar Sonar Navig. 6(9), 822-833 (2012)

25. J. Liang, H. C. So, J. Li, et al., Unimodular sequence design based on alternating direction method of multipliers. IEEE Trans. Sig. Proc. 64(20), 5367-5381 (2016)

26. D. J. Kershaw, R. J. Evans, Optimal waveform selection for tracking systems. IEEE Trans. Inf. Theory. 40, 1536-1550 (1994)

27. D. J. Kershaw, R. J. Evans, Waveform selective probabilistic data association. IEEE Trans. Aerosp. Electron. Syst. 33, 1180-1188 (1997)

28. C. Rago, P. Willett, Y. Bar-Shalom, Detection-tracking performance with combined waveforms. IEEE Trans. Aerosp. Electron. Syst. 34, 612-624 (1998)

29. R. Niu, P. Willett, Y. Bar-Shalom, Tracking considerations in selection of radar waveform for range and range-rate measurements. IEEE Trans. Aerosp. Electron. Syst. 38, 467-487 (2002)

30. S. D. Howard, S. Suvorova, W. Moran, in Int. Conf. Waveform Diversity and Design, Waveform libraries for radar tracking applications, (Edinburgh, 2004). https://doi.org/10.1109/iwddc.2004.8317579

31. S. M. Hong, R. J. Evans, H. S. Shin, Optimization of waveform and detection threshold for range and range-rate tracking in clutter. IEEE Trans. Aerosp. Electron. Syst. 41(1), 17-33 (2005)

32. B. F. L. Scala, W. Moran, R. J. Evans, Optimal adaptive waveform selection for target detection. Proc. Int. Conf. Radar, 492-496 (2003). https://doi.org/10.1109/radar.2003.1278791

33. S. P. Sira, A. Papandreou-Suppappola, D. Morrell, Dynamic configuration of time-varying waveforms for agile sensing and tracking in clutter. IEEE Trans. Sig. Proc. 55(7), 3207-3217 (2007)

34. R. Calderbank, S. D. Howard, B. Moran, Waveform diversity in radar signal processing. IEEE Sig. Proc. Mag. 26(1), 32-41 (2009)

35. J. J. Benedetto, I. Konstantinidis, M. Rangaswamy, Phase-coded waveforms and their design. IEEE Sig. Proc. Mag. 26(1), 22-31 (2009)

36. M. Golay, Complementary series. Inf. Theory Ire Trans. 7(2), 82-87 (1961)

37. Y. Li, W. B. Chu, More Golay sequences. IEEE Trans. Inf. Theory. 51(3), 1141-1145 (2005)

38. M. G. Parker, K. G. Paterson, T. Chintha, in Wiley Encyclopedia of Telecommunications. ed. by J. G. Proakis, Golay Complementary Sequences (Wiley, 2003)

39. A. Pezeshki, A. R. Calderbank, W. Moran, et al., Doppler resilient Golay complementary waveforms. IEEE Trans. Inf. Theory. 54(9), 4254-4266 (2008)

40. T. Hayashi, Zero-correlation zone sequence set constructed from a perfect sequence and a complementary sequence pair. IEICE Trans. Fundam. Electron. Commun. Comput. Sci. 91(7), 1676-1681 (2010)

41. J. Song, P. Babu, D. P. Palomar, Sequence set design with good correlation properties via majorization-minimization. IEEE Trans. Sig. Proc. 64(11), 2866-2879 (2016)

42. M. Alaee-Kerahroodi, M. R. Bhavani Shankar, K. V. Mishra, B. Ottersten, in 201920 th International Radar Symposium (IRS), Meeting the lower bound on designing set of unimodular sequences with small aperiodic/periodic ISL, (UIm, Germany, 2019), pp. 1-13. https://doi.org/10.23919/irs.2019.8768085

43. M. Alaee-Kerahroodi, M. Modarres-Hashemi, M. M. Naghsh, Designing sets of binary sequences for MIMO radar systems. IEEE Trans. Sig. Proc. 67(13), 3347-3360 (2019)

44. M. Alaee-Kerahroodi, S. Sedighi, M. R. B. Shankar, B. Ottersten, in IEEE International Conference on Acoustics, Speech and Signal Processing (ICASSP), Designing (In)finite-alphabet sequences via shaping the radar ambiguity function, (Brighton, 2019), pp. 4295-4299. https://doi.org/10.1109/icassp.2019.8682216

\section{Publisher's Note}

Springer Nature remains neutral with regard to jurisdictional claims in published maps and institutional affiliations. 